\title{
Chronic parasitization by Nosema microsporidia causes global expression changes in core nutritional, metabolic and behavioral pathways in honey bee workers (Apis mellifera)
}

Holly L Holt ${ }^{1,3^{*}}$, Katherine A Aronstein ${ }^{2}$ and Christina M Grozinger ${ }^{1}$

\begin{abstract}
Background: Chronic infections can profoundly affect the physiology, behavior, fitness and longevity of individuals, and may alter the organization and demography of social groups. Nosema apis and Nosema ceranae are two microsporidian parasites which chronically infect the digestive tract of honey bees (Apis mellifera). These parasites, in addition to other stressors, have been linked to increased mortality of individual workers and colony losses in this key pollinator species. Physiologically, Nosema infection damages midgut tissue, is energetically expensive and alters expression of immune genes in worker honey bees. Infection also accelerates worker transition from nursing to foraging behavior (termed behavioral maturation). Here, using microarrays, we characterized global gene expression patterns in adult worker honey bee midgut and fat body tissue in response to Nosema infection.

Results: Our results indicate that $N$. apis infection in young workers ( 1 and 2 days old) disrupts midgut development. At 2 and 7 days post-infection in the fat body tissue, N. apis drives metabolic changes consistent with energetic costs of infection. A final experiment characterizing gene expression in the fat bodies of 14 day old workers parasitized with N. apis and N. ceranae demonstrated that Nosema co-infection specifically alters conserved nutritional, metabolic and hormonal pathways, including the insulin signaling pathway, which is also linked to behavioral maturation in workers. Interestingly, in all experiments, Nosema infection did not appear to significantly regulate overall expression of canonical immune response genes, but infection did alter expression of acute immune response genes identified in a previous study. Comparative analyses suggest that changes in nutritional/metabolic processes precede changes in behavioral maturation and immune processes.
\end{abstract}

Conclusions: These genome-wide studies of expression patterns can help us disentangle the direct and indirect effects of chronic infection, and understand the molecular pathways that regulate disease symptoms.

Keywords: Nosema, Honey bee, Behavioral maturation, Insulin signaling pathway, Innate immunity, Chronic parasite, Nutrition, Genomics, Stress

\footnotetext{
* Correspondence: hlh192@psu.edu

'Department of Entomology, Center for Pollinator Research, Center for Chemical Ecology, Huck Institutes of the Life Sciences, Pennsylvania State University, University Park, USA

${ }^{3} 3 \mathrm{~A}$ Chemical Ecology Laboratory, Pennsylvania State University, Orchard Road, University Park, PA 16802, USA

Full list of author information is available at the end of the article
} 


\section{Background}

Chronic parasitization can have profound effects on host physiology, behavior and fitness [1]. Studying the molecular mechanisms mediating host responses to prolonged parasitism can be challenging, since chronic infections can lead to complex and extensive downstream effects. Thus, the direct and indirect effects of parasites, and how these contribute to symptoms of infection, can be difficult to disentangle. In social systems, the interactions between parasites and host defense strategies may lead to changes in host social interactions and organization, which in turn can alter transmission patterns of the parasite and have effects on the viability of the social group [2]. The microsporidian parasites Nosema apis and Nosema ceranae and their honey bee (Apis mellifera) hosts [3] provide an excellent model for studying the molecular basis of direct and indirect effects of chronic parasitization on individuals, social interactions and social group organization.

Microsporidia are a specialized group of derived, pathogenic fungi that cause chronic, intracellular infections, generally in animal hosts [4]. In honey bees, environmental spores of Nosema spp. are spread via fecal/oral routes. Once ingested, spores germinate, infecting host midgut cells, and parasites replicate intracellularly as vegetative states [5]. Ultimately, new spores are produced which may infect other host cells or are voided [3]. Crowded hive environments can facilitate rapid pathogen transmission which may be counteracted by individuals' immune systems, social immune defenses and the genetic diversity within a colony, fostered by the polyandrous behavior of queens [6]. Despite individual and social defenses, infection with either species of Nosema may undermine colony health. However, there is global variation in reports of species virulence in cage trials and field studies. Cage studies comparing species virulence offer mixed support for greater $N$. ceranae virulence contrasted with $N$. apis [7-10] while $N$. ceranae in particular has been regionally correlated with colony morbidity and mortality (see [3,11] for a review and [12]). A number of factors, including experimental conditions, the presence of other stressors (e.g. pesticides, other diseases), temperature/climate and potentially Nosema control agents [13-19], are thought to modulate virulence and/or distribution of either parasite species, which may partially explain global patterns in Nosema spp. prevalence and variation in experimental outcomes. Other species-specific differences in parasite pathology, such as differences in replication rate and damage done to midgut tissue, in addition to molecular evidence (which remains to be verified via microscopy approaches) for differences in host tissue distribution, may also contribute to $N$. ceranae's alleged greater virulence $[8,14,15,20,21]$. By studying gene expression patterns in tissues that are directly and systemically affected by Nosema species parasitization, we can begin to unravel the causative pathways that underlie host morbidity and mortality.

At the individual level, Nosema infection is energetically costly which may, in part, drive reported physiological and behavioral symptoms of infection in workers. Diverse studies conducted in cages and/or the field have documented infection costs. Because microsporidia are specialized pathogens with reduced metabolic capacities, they rely heavily on their hosts to furnish energy for parasite growth and reproduction [4] as indicated by the recently published Spartan genome of N. ceranae [22]. Indeed, $N$. ceranae infected workers are energetically impoverished: they are hungrier and more susceptible to starvation than controls [23,24], have diminished hemolymph trehalose concentrations [25] and are less likely to feed nestmates via trophallaxis [26]. N. apis similarly disrupts worker nutrition and energy balance. Infected workers have altered titers of hemolymph amino acids [27]. However, one study directly comparing energetic costs across parasite species suggests that $N$. apis infection is not as energetically draining as $N$. ceranae infection [24]. More studies directly comparing energetic costs of each parasite, with longer time-frames to accommodate the chronic nature of infection are needed. Comparative functional analysis of parasite genomes may also help to explain species-specific differences in energetic costs.

Symptoms of energetic distress in infected bees are not surprising given that both species of Nosema destructively replicate within honey bee midgut tissue $[8,20,28,29]$ which likely impairs their hosts' ability to digest food and/or absorb nutrients. Within midgut cells, $N$. apis and $N$. ceranae associate with host cell mitochondria $[8,30]$ (consistent with a general microsproidian strategy for obtaining host energy [4]) and RNAi studies indicate that $N$. ceranae commandeers host ATP through ATP/ADP transporters [31], further burdening host metabolism. Other physiological symptoms of infection include altered immune function. One study, by monitoring expression of select immune genes, has indicated that $N$. ceranae but not $N$. apis may immunosuppress workers [32] at 7 days post-infection. However, immune gene expression patterns in response to $N$. ceranae infection are modulated by initial spore dosage and disease incubation period [33].

Infection with either species of Nosema alters worker behavior by accelerating worker transition from nursing to foraging activities, a process termed behavioral maturation [34-38]. The molecular and physiological mechanisms by which Nosema infection accelerates worker behavioral maturation have not been fully characterized. Workers begin their adult lives with the in-hive task of brood care, shifting to other duties such as queen attendance or nest guarding and finally to foraging as they age [39]. The transition from nursing to foraging behavior is 
driven/accompanied by large-scale, internal changes in worker nutrition, metabolism, hormonal profile and physiology $[39,40]$ in addition to external signals from the colony, including pheromones released by the brood, workers and queen [41]. Compared to nurse bees, foragers have lower lipid stores in their abdominal fat body tissue [42], decreased expression of genes related to lipid and protein metabolism, and increased expression of genes related carbohydrate metabolism [43]. Consequently, inhibition of fat accumulation can accelerate worker transition to a forager state [44] and poor diet in young adult workers changes expression of some genes to a forager-like profile [43]. Foragers also have higher levels of juvenile hormone $(\mathrm{JH})$ and lower levels of the storage protein vitellogenin $(\mathrm{Vg})$, which negatively interacts with $\mathrm{JH}$ to mediate behavioral maturation [45]. Artificially increasing $\mathrm{JH}$ titers [46] or decreasing $\mathrm{Vg}$ levels can accelerate the transition to foraging $[47,48]$. Importantly, the $\mathrm{JH} / \mathrm{Vg}$ regulatory pathway interacts with worker nutritional status and the insulin signaling pathway, which has also been linked to behavioral maturation [49-51].

Nosema may accelerate behavioral maturation in workers by modifying host nutritional, metabolic and hormonal attributes. Nosema broadly and negatively impacts worker energetic status which may drive changes in behavioral maturation [23-25]. Studies have also found mixed effects of $N$. ceranae on worker expression levels of vitellogenin $(v g)$ and circulating $\mathrm{JH}$ titers. While one study has reported negligible impact of $N$. ceranae on caged worker $v g$ expression at several times post-infection [33], others have found that infection reduces $v g$ expression at 7 days post-infection [32] (which is consistent with precocious foraging) or causes aberrant patterns of $v g$ expression in caged workers, but no difference in expression between workers housed in colonies [36]. Also, JH titers are elevated in workers infected with $N$. ceranae and $N$. apis (consistent with earlier foraging), however, the degree to which $\mathrm{JH}$ titers are raised varies with Nosema species and with parasite strain [36,52].

Because worker nutritional, metabolic and hormonal pathways interact [40], it is unclear if: (1) Nosema precipitates behavioral maturation by modulating expression of genes that regulate behavioral maturation, and/or (2) Nosema imposes such stringent metabolic costs that host nutritional status is diminished, driving changes in metabolic or hormonal processes that regulate maturation, and/or (3) Nosema infection interferes with nutrient uptake, resulting in cascading effects on metabolism and behavioral maturation. In addition, because a large number of physiological changes normally accompany behavioral maturation, it is difficult to determine whether other symptoms of Nosema infection arise from pathological changes mediated directly by infection versus changes mediated by accelerated development. For example, changes in worker immune function are linked to worker behavioral state which correlates with age $[53,54]$.

Here, we used whole genome microarrays to monitor global gene expression patterns in honey bee worker midgut and fat body tissue and to identify molecular processes associated with the symptoms of Nosema infection. We characterized gene expression in midgut tissue infected with $N$. apis at 1 and 2 days post-infection (pi) and infected fat body tissue at 2 and 7 days pi. We also examined gene expression in co-infected ( $N$. apis and $N$. ceranae) fat body tissue at 14 days pi. We sought to capture early, local immune responses in midgut tissue, the initial site of infection. We also investigated immune, hormonal and metabolic changes in fat body tissue which is an important regulator of insect nutrient stores, development and systemic immunity $[55,56]$. We compared our lists of genes significantly regulated by infection to: a list of genes significantly regulated by $N$. ceranae infection in worker midgut tissue [28], genes associated with canonical immune responses [57], genes associated with acute responses to general immune challenges [58], genes associated with worker behavioral maturation, and genes associated with robust or restrictive diets [43]. Though these studies do not allow us to separate speciesspecific effects of Nosema infection, the results from these experiments provide a holistic view of how Nosema impacts gene expression in these worker tissues. Taken together, these findings allow us to better understand the molecular mechanisms regulating host-parasite interactions in this system, and begin to evaluate interactions between Nosema infection and host nutrition, physiology and behavioral maturation.

\section{Results}

\section{Effects of Nosema apis infection on midgut and fat body gene expression}

PCR of midgut tissue confirmed that samples collected in 2008 (midguts collected from bees 1, 2 and 7-days postinfection) had $N$. apis infections only (Additional file 1: Figure S1A). We used microarrays to characterize global gene expression in the midguts collected 1 and 2 days post-infection (pi) and fat bodies collected 2 and 7 days pi. After removing transcripts with expression levels below background, 11,746 and 12,492 transcripts for midgut and fat body tissues were retained for further analysis. Transcripts with significant expression differences (incorporating transcripts with significant age, treatment and age $\mathrm{x}$ treatment effects) for each tissue were determined using a multivariate ANOVA. We identified 736 and 2,343 unique significantly, differentially expressed transcripts in midgut and fat body tissues respectively (FDR $<0.001$, Additional file 2: Table S1 and Additional file 2: Table S2).

Principal components analysis (Figure 1) revealed the effects of treatment and age on gene expression patterns 


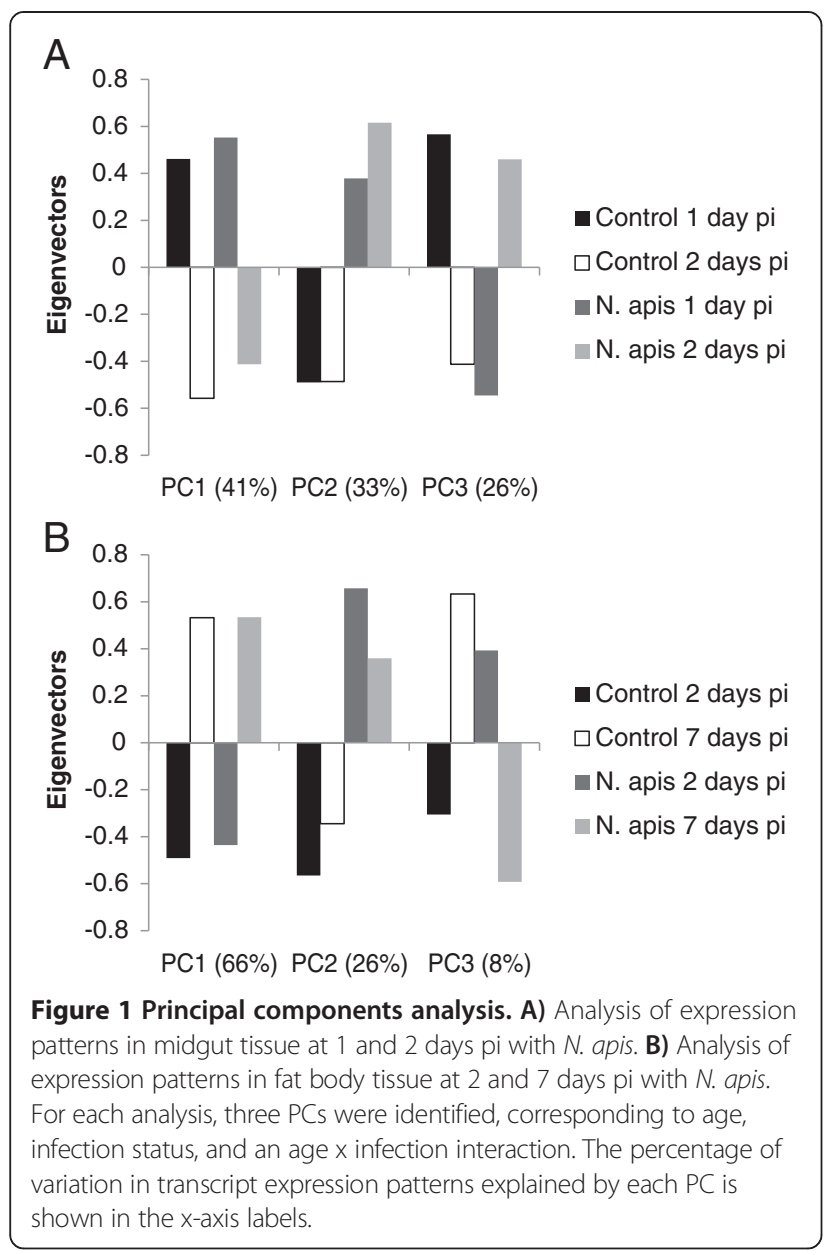

in the two tissues. For the midguts: (1) age explained $41 \%$ of the variability, (2) treatment explained $33 \%$ of the variability and, (3) an age $\mathrm{x}$ treatment interaction explained $26 \%$ of the variability (Figure $1 \mathrm{~A}$ ). For the fat bodies: (1) age explained $66 \%$ of the variability (2) treatment explained $26 \%$ of the variability and (3) an age $x$ treatment interaction explained the remaining $8 \%$ of the variability (Figure 1B).

For subsequent analyses, we selected the subset of transcripts that were regulated by $N$. apis infection only (not by age or an age $\mathrm{x}$ treatment interaction). We found that 150 and 278 transcripts were regulated by treatment only in the midgut and fat body tissues (Additional file 2: Table S1 and Additional file 2: Table S2). Gene ontology (GO) analysis of the 150 midgut transcripts (corresponding to 84 unique Drosophila orthologs) yielded over-represented clusters of genes involved in tube morphogenesis, regulation of neurogenesis, sensory perception of chemical stimuli and multicellular organismal processes $(p, 0.05$, Table 1$)$. Analysis of the 278 fat body transcripts (corresponding to 201 unique Drosophila orthologs) yielded over-represented clusters of genes involved in metabolic processes, mitochondrial membrane organization, lipid metabolic processes, gene expression, ncRNA processing, and regulation of apoptosis $(p<0.05$, Table 1$)$.

We also examined directional expression of the transcripts from significant GO categories. In midgut tissue, Nosema apis infection increased expression of transcripts involved in regulation of neurogenesis, tube morphogenesis, and multicellular organismal processes. The opposite pattern was observed for sensory perception of chemical stimulus (Additional file 1: Figure S2A). In fat body tissue, there were 2-10 times more transcripts upregulated in controls vs infected bees for primary metabolic processes, gene expression, ncRNA processing, regulation of apoptosis and mitochondrial membrane organization. The opposite pattern was only observed for lipid metabolic process (Additional file 1: Figure S2B).

\section{Effects of mixed Nosema apis and Nosema ceranae infection on fat body gene expression}

Given that $N$. ceranae has achieved global prevalence and that co-infections in single bees occur naturally $[14,59]$, and also considering the chronic nature of microsporidian pathology, we conducted a second array study using $N$. apis and $N$. ceranae co-infected bees at a later time-point (14 days). While we are unable to directly compare impact of co-infection at this later time point to effects of $N$. apis infection at earlier time points in fat body tissue, together these studies provide insights into how microsporidian infection globally impacts worker metabolism.

PCR of midgut tissues confirmed that samples collected in 2010 were co-infected (Additional file 1: Figure S1B). We processed array data as before. After removing transcripts with expression levels below background, we included 12,596 transcripts in the analysis. A multivariate ANOVA identified 1,447 transcripts that were significantly, differentially regulated by Nosema co-infection (FDR < 0.0001; Additional file 2: Table S3).

GO analysis of these 1,447 transcripts (corresponding to 1,015 unique Drosophila orthologs) yielded overrepresented clusters involved in metabolism, cell organization and transport, development and immunity (Table 2). We determined the directional impact of infection on gene expression for transcripts included in significant GO categories. There were 3-17 times more transcripts that were upregulated in controls compared with those upregulated in infected bees within each significant $\mathrm{GO}$ category related to cellular organization and transport and metabolism. Only transcripts relating to immunity did not show strong trends in directional expression with equal numbers upregulated by both treatments (Additional file 1: Figure S2C). 
Table 1 Functional analysis of transcripts regulated by $\mathbf{N}$. apis infection

\begin{tabular}{|c|c|c|c|}
\hline Tissue and timepoint (days pi) & GO term & GO biological process & $p$-value \\
\hline \multirow[t]{4}{*}{ Midgut $(1,2)$} & GO:0035239 & Tube morphogenesis & $9.19 \mathrm{E}-03$ \\
\hline & GO:0050767 & Regulation of neurogenesis & $3.58 \mathrm{E}-02$ \\
\hline & GO:0007606 & Sensory perception of chemical stimulus & $3.42 \mathrm{E}-02$ \\
\hline & GO:0032501 & Multicellular organismal process & 4.99E-02 \\
\hline \multirow[t]{6}{*}{ Fat body $(2,7)$} & GO:0044238 & Primary metabolic process & 3.65E-03 \\
\hline & GO:0007006 & Mitochondrial membrane organization & $1.02 \mathrm{E}-02$ \\
\hline & GO:0006629 & Lipid metabolic process & $2.59 \mathrm{E}-02$ \\
\hline & GO:0010467 & Gene expression & $2.62 \mathrm{E}-02$ \\
\hline & GO:0034470 & ncRNA processing & $2.70 \mathrm{E}-02$ \\
\hline & GO:0042981 & Regulation of apoptosis & $3.44 \mathrm{E}-02$ \\
\hline
\end{tabular}

Transcripts significantly regulated by infection in worker midgut and fat body tissue were selected for functional analysis and overrepresented GO processes were identified.

\section{Comparisons with previous genomic studies}

Dussaubat and colleagues (2012) identified 336 genes that were significantly regulated by $N$. ceranae in worker midgut tissue at 7 days pi (14 days old) [28]. Only 6 of these genes were also found to be regulated in midgut tissue in our study. This relatively small overlap is likely due to differences in inoculation time, inoculum dosage and species of Nosema used in these studies. However, examination of GO analyses revealed that both studies produced overrepresented biological clusters involved in midgut development, including genes related to neuronal and tracheal processes and midgut structure. In our study, genes involved in "regulation of neurogenesis" were affected while genes involved in "neuron differentiation", "neuron development" and "axonogenesis" were regulated in Dussaubat et al. (2012). Also, in our study, genes related to "tube morphogenesis" were affected while genes involved in "open tracheal system development" and "morphogenesis of an epithelium" were regulated in Dussaubat et al. (2012).

Evans and colleagues identified canonical insect immune genes from the honey bee genome [57]. Of these, 166 genes were included on the array platform, which encompassed mediators of the major immune response pathways including Toll, IMD and JAK/Stat. No significant overlap (Hypergeometric Test; $p>0.05$ ) was observed between any of our Nosema regulated transcript lists and the list of canonical honey bee immune genes (Table 3). We also compared our significantly regulated transcripts with a list of "acute" immune response transcripts regulated in the fat bodies of worker honey bees injected with saline, Sephadex beads or dead E. coli bacteria (evaluated at 6 hours pi) [58]. There was significant overlap with all three Nosema-regulated transcript lists (Table 3;

Table 2 Functional analysis of transcripts regulated by co-infection

\begin{tabular}{|c|c|c|c|}
\hline Functional category & GO term & GO biological process & $p$-value \\
\hline \multirow[t]{7}{*}{ Metabolism } & GO:0042180 & Cellular ketone metabolic process & $2.42 \mathrm{E}-04$ \\
\hline & GO:0006520 & Cellular amino acid metabolic process & 3.77E-04 \\
\hline & GO:0009152 & Purine ribonucleotide biosynthetic process & $1.71 \mathrm{E}-03$ \\
\hline & GO:0044262 & Cellular carbohydrate metabolic process & $1.72 \mathrm{E}-02$ \\
\hline & GO:0006629 & Lipid metabolic process & $2.31 \mathrm{E}-02$ \\
\hline & GO:0042440 & Pigment metabolic process & $4.58 \mathrm{E}-02$ \\
\hline & GO:0008152 & Metabolic process & 4.64E-02 \\
\hline \multirow[t]{4}{*}{ Cell organization and transport } & GO:0051234 & Establishment of localization & $3.56 \mathrm{E}-04$ \\
\hline & GO:0006811 & Ion transport & $5.32 \mathrm{E}-04$ \\
\hline & GO:0016192 & Vesicle-mediated transport & $2.10 \mathrm{E}-02$ \\
\hline & GO:0033227 & dsRNA transport & $3.52 \mathrm{E}-02$ \\
\hline \multirow[t]{2}{*}{ Other } & GO:0002376 & Immune system process & $1.99 \mathrm{E}-02$ \\
\hline & GO:0046664 & Dorsal closure, amnioserosa morphology change & $2.28 \mathrm{E}-02$ \\
\hline
\end{tabular}

Transcripts significantly regulated by Nosema co-infection in worker fat body tissue were selected for functional analysis and overrepresented GO processes were identified. 
Table 3 Overlap of Nosema spp. regulated transcripts with canonical and acute immune response transcripts

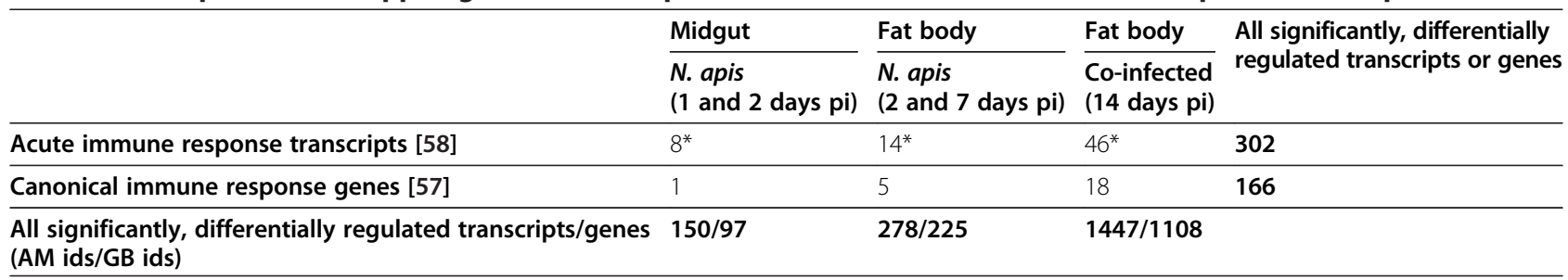

*Indicates significantly more overlap than expected by chance (Hypergeometric Test; $p<0.02$ at most).

Study results were compared with data from [58] and [57].

Hypergeometric Test; $p<0.020$ for midguts, $p<0.004$ and $p<0.010$ for fat bodies at 2 and 7 days pi and 14 days pi respectively).

Finally, Ament and collaborators identified differentially expressed transcripts in the fat bodies of nurses versus foragers and workers fed on rich (pollen and honey) versus poor (sugar syrup) diets [43]. Significantly more overlap than expected by chance was observed between the diet transcript list and genes differentially regulated in the fat bodies of $N$. apis infected ( 2 and 7 days pi) and co-infected (14 days pi) workers (Hypergeometric Test; $\mathrm{p}<0.009$ and $\mathrm{p}<<0.0001$ respectively; Table 4). Significantly more overlap than expected by chance was observed with the behavioral maturation (nurse vs forager) transcripts only with transcripts differentially expressed in the fat bodies of co-infected 14 day pi bees (Hypergeometric Test: $\mathrm{p}<<0.0001$; Table 4).

Since overlap was strongest with diet and behavioral maturation transcript lists for co-infected samples (14 days pi), we next examined directional overlap among these sets of transcripts (Table 5). Notably, there was significantly more overlap than expected by chance across transcripts that were upregulated in controls and nurses, or bees fed on rich diets. There was also significantly more overlap than expected by chance across transcripts that were upregulated in Nosema co-infected bees and foragers, or bees fed on poor diets. Conversely, there was either no more overlap than expected by chance or significantly less overlap than expected by chance across controls and foragers, or bees fed on poor diets and across Nosema co-infected bees and nurses, or bees fed on rich diets. A Chi-Square test revealed that across studies, patterns of gene expression between controls and co-infected bees were more similar to expression patterns of bees fed on rich and poor diets respectively than to nurses and foragers $\left(\mathrm{X}^{2}=93.72, p<0.001\right)$.

$\mathrm{GO}$ analysis of genes with the same directional overlap (203 unique Drosophila orthologues) for nurses and controls and foragers and infected bees yielded significant clusters $(p<0.05)$ related to metabolism and one cluster that was nearly significant related to immunity $(\mathrm{p}=0.051)$ (Table 6). GO analysis of genes with the same directional overlap (417 unique Drosophila orthologues) for bees fed on a rich diet and controls and bees fed on a poor diet and infected bees yielded significant clusters $(p<0.05)$ related to metabolism, cellular organization and immunity (Table 7).

\section{Candidate genes}

Based on results from GO analyses and study comparisons, we selected candidate genes associated with metabolism, behavioral maturation and immunity that were significantly, differentially regulated in control versus co-infected workers at 14 days pi. Where data was available, we determined whether candidate genes were also upregulated in nurses versus foragers, and bees fed on rich versus poor diets [43]. Results of pairwise contrasts are summarized in Table 8 . Selected genes were not necessarily significantly, differentially regulated in all three studies. Where differential expression was observed, a number of genes tracked directional expression expectations (e.g. upregulated across controls, nurses and/or bees fed on rich diets or were upregulated across co-infected bees, foragers and bees fed on poor diets). Other genes showed infection status by behavioral state (nurse vs. forager) or diet (rich vs. poor diet) interactions.

Table 4 Overlap of Nosema spp. regulated transcripts with behavioral state and diet lists

\begin{tabular}{lllll}
\hline & Fat body & Fat body & $\begin{array}{l}\text { All significantly, differentially } \\
\text { regulated transcripts }\end{array}$ \\
\cline { 2 - 3 } & N. apis (2 and 7 days pi) & Co-infected (14 days pi) & $\mathbf{2 6 4 0}$ \\
\hline Nurse vs forager & 52 & $87^{*}$ & $654^{*}$ & 3351 \\
\hline Rich vs poor diet & 278 & $\mathbf{1 4 4 7}$ & \\
\hline
\end{tabular}

*Indicates significantly more overlap than expected by chance (Hypgeometric Test; $p<0.009$ at most).

Study results were compared with data from [43]. 
Table 5 Directional overlap of transcripts regulated by Nosema co-infection, worker behavioral state and diet

\begin{tabular}{lllll}
\hline Transcript list & $\begin{array}{l}\text { Upregulated in } \\
\text { co-infected bees } \\
\text { (577 transcripts) }\end{array}$ & $\begin{array}{l}\text { Representation factor, Upregulated in controls } \\
\text { ( } \text {-value) }\end{array}$ & $\begin{array}{l}\text { Representation factor, } \\
\text { (870 transcripts) }\end{array}$ \\
\hline Upregulated in nurses (1204 transcripts) & 30 & $0.6,(p<0.0003)$ & 151 & $1.9,(p<<0.0001)$ \\
\hline Upregulated in foragers (1436 transcripts) & 149 & $2.4,(p<<0.0001)$ & 95 & $1.0,(p<0.4260)$ \\
\hline $\begin{array}{l}\text { Upregulated in workers fed on a rich diet } \\
\text { (1492 transcripts) }\end{array}$ & 13 & $0.2,(p<<0.0001)$ & 342 & $3.5,(p<<0.0001)$ \\
\hline $\begin{array}{l}\text { Upregulated in workers fed on a poor diet } \\
\text { (1859 transcripts) }\end{array}$ & 265 & $3.3,(p<<0.0001)$ & 34 & $0.3,(p<<0.0001)$ \\
\hline
\end{tabular}

Transcripts upregulated in controls or by Nosema co-infection (14 days pi) were overlapped with transcripts upregulated by worker behavioral state or diet treatments [43]. Significant overlap between transcript lists was identified using hypergeometric tests. A representation factor equal to one indicates no more overlap than expected by chance, a factor greater than one indicates more overlap than expected by chance, and a factor less than one indicates less overlap than expected by chance.

Some of these candidate genes are associated with the immune system. For example, we found that Nosema coinfection regulated expression of members (pelle, ECSIT) of the Toll-like receptor signaling cascade, which is thought to mobilize insect immune effectors in response to challenge with fungi or gram + bacteria [56]. We also observed changes in domeless which is a central receptor in the JAK/Stat pathway [56].

Several of these genes are involved in nutritional and metabolic pathways. Nosema co-infection regulated expression of several members of the insulin signaling pathway (Apis mellifera ilp-2; Akt1; Pdk1) in addition to downstream transcription factors (forkhead box, sub-group O) [60]. As previously discussed, the insulin signaling pathway has been implicated in mediating worker behavioral maturation [50]. Co-infection also affected expression of gigas, which produces the Tsc2 protein in the Drosophila insulin signaling pathway. TSC2 links insulin signaling to the related TOR pathway which coordinates cell size and growth [61]. In honey bees, initial studies indicate that changes in TOR signaling may also affect behavioral maturation [50]. We also saw significant regulation of venus kinase receptor $(v k r)$ which belongs to a recently discovered, nutrient-sensitive family of receptor tyrosine kinase proteins found in some invertebrates. Expression of $v k r$ is correlated with development in male honey bees and reproduction in other model invertebrates [62]. As previously discussed, abdominal lipid content is an important physiological factor that contributes to worker behavioral maturation $[42,44]$. Co-infection regulated several genes involved in lipid metabolism (lipase-1, lipid storage droplet 2; carnitine O-palmitoyl transferase 1) that have been previously correlated with worker behavioral state and/or diet [43]. Interestingly, $l s d-2$ expression is also modulated by acute infections [58].

Co-infection upregulated expression of ultraspiracle (usp), a transcription factor that responds to $\mathrm{JH}$ titers and that may act in concert with other factors to regulate behavioral maturation. For example, usp knockdown slows onset of foraging behavior [63]. Co-infection also modified expression of juvenile hormone esterase (Jhe) which likely breaks down JH in worker honey bees [64] in addition to expression of juvenile hormone expoxide hydrolase (Jheh). Previous studies suggest the Jheh does not break down $\mathrm{JH}$ in honey bees as in other insects, but is responsive to worker behavioral state and diet $[43,65]$.

Validation of gene expression results using quantitative real-time PCR

Given that the number of canonical immune response genes regulated by Nosema infection was low, we used qRT-PCR to examine expression levels of five candidate

Table 6 Functional analysis of transcripts regulated by Nosema co-infection and worker behavioral state

\begin{tabular}{|c|c|c|c|}
\hline Functional category & GO term & GO biological process & $p$-value \\
\hline \multirow[t]{7}{*}{ Metabolism } & GO:0042180 & Cellular ketone metabolic process & $3.40 \mathrm{E}-04$ \\
\hline & GO:0034754 & Cellular hormone metabolic process & $3.27 \mathrm{E}-03$ \\
\hline & GO:0008152 & Metabolic process & $6.10 \mathrm{E}-03$ \\
\hline & GO:0006189 & 'De novo' IMP biosynthetic process & $7.81 \mathrm{E}-03$ \\
\hline & GO:0006629 & Lipid metabolic process & $1.59 \mathrm{E}-02$ \\
\hline & GO:0006066 & Alcohol metabolic process & $1.65 \mathrm{E}-02$ \\
\hline & GO:0032787 & Monocarboxylic acid metabolic process & $2.34 \mathrm{E}-02$ \\
\hline Other & GO:0006955 & Immune response* & 5.05E-02 \\
\hline
\end{tabular}

*Not significant at $p<0.05$.

Significantly regulated transcripts in the fat body tissue of co-infected workers (14 days pi) were overlapped with transcripts significantly regulated in the fat bodies of nurses and foragers [43]. Transcripts that were upregulated in controls and nurses or upregulated in infected bees and foragers were selected for functional analysis and overrepresented GO processes were identified. 
Table 7 Functional analysis of transcripts regulated by Nosema co-infection and worker diet

\begin{tabular}{|c|c|c|c|}
\hline Functional category & GO term & GO biological process & $p$-value \\
\hline \multirow[t]{11}{*}{ Metabolism } & GO:0009152 & Purine ribonucleotide biosynthetic process & 3.46E-06 \\
\hline & GO:0006082 & Organic acid metabolic process & 7.30E-05 \\
\hline & GO:0008152 & Metabolic process & $1.22 \mathrm{E}-03$ \\
\hline & GO:0009064 & Glutamine family amino acid metabolic process & $3.38 \mathrm{E}-03$ \\
\hline & GO:0051186 & Cofactor metabolic process & 1.07E-02 \\
\hline & GO:0044262 & Cellular carbohydrate metabolic process & $1.71 \mathrm{E}-02$ \\
\hline & GO:0032787 & Monocarboxylic acid metabolic process & $1.76 \mathrm{E}-02$ \\
\hline & GO:0009167 & Purine ribonucleoside monophosphate metabolic process & $2.36 \mathrm{E}-02$ \\
\hline & GO:0005996 & Monosaccharide metabolic process & $2.80 \mathrm{E}-02$ \\
\hline & GO:0009056 & Catabolic process & $3.92 \mathrm{E}-02$ \\
\hline & GO:0006119 & Oxidative phosphorylation & 4.46E-02 \\
\hline \multirow[t]{3}{*}{ Cellular organization } & GO:0051234 & Establishment of localization & $9.03 \mathrm{E}-03$ \\
\hline & GO:0045184 & Establishment of protein localization & $2.14 \mathrm{E}-02$ \\
\hline & GO:0016192 & Vesicle-mediated transport & $3.20 \mathrm{E}-02$ \\
\hline \multirow[t]{4}{*}{ Immunity and other } & GO:0006955 & Immune response & $2.08 \mathrm{E}-02$ \\
\hline & GO:0008063 & Toll signaling pathway & $2.72 \mathrm{E}-02$ \\
\hline & GO:0050777 & Negative regulation of immune response & $3.08 \mathrm{E}-02$ \\
\hline & GO:0050770 & Regulation of axonogenesis & 4.56E-02 \\
\hline
\end{tabular}

Significantly regulated transcripts in the fat body tissue of co-infected workers (14 days pi) were overlapped with transcripts significantly regulated in the fat bodies of workers fed on rich and poor diets [43]. Transcripts that were upregulated in controls and workers fed on rich diets or upregulated in infected bees and workers fed on poor diets were selected for functional analysis and overrepresented GO processes were identified.

genes with immune (abaecin, hymenoptaecin, defensin-1), nutritional and/or developmental functions (vitellogenin, hexamerin 70b) across treatment groups for RNA extracted from fat body tissue at 7 and 14 days. Gene expression was standardized to actin. Results indicated that all genes at 7 days pi followed a general trend for upregulation in controls relative to $N$. apis treated bees, however none of these trends were significant (MannWhitney U Test, $\mathrm{p}>0.05$; Additional file 1: Figure S3A). Results at 14 days pi (co-infected bees) revealed a more heterogeneous pattern of expression though no differences in expression were significant across treatment groups (Mann-Whitney U Test, $\mathrm{p}>0.05$; Additional file 1 : Figure S3B). We also verified that no corresponding transcripts for these genes were significantly differentially expressed on the microarrays (Additional file 2: Table S4).

\section{Discussion}

Gene expression analysis suggests that infection with Nosema apis has immediate, deleterious consequences for midgut structure and function. Resulting tissue damage and subsequent siphoning of host resources may significantly and negatively impact metabolic and nutritional pathways in worker fat body tissue. Ultimately, for chronologically advanced co-infections, metabolic and nutritional changes may affect expression of genes linked to worker behavioral maturation, predisposing infected individuals to foraging tasks. Altered expression of these genes may also simultaneously drive changes in worker immune function, though it remains to be determined if immune pathways are altered by nutritional deprivation, behavioral maturation, increasing amounts of tissue damage and/or directly by infection.

Importantly, when synthesizing results across studies, our findings must be interpreted with caution due to different time points and species of infection used in these three microarray analyses. In addition, by caging study subjects in these experiments we strongly controlled worker environment and standardized food access. This reduced variability in gene expression, facilitating identification of Nosema-mediated changes. However, as individual worker nutritional and behavioral status are closely linked to colony signals and resources, future studies examining gene expression in workers housed in colonies are needed to validate findings from this study. Finally, additional studies employing pure $N$. apis and $N$. ceranae infections may also underscore similarities and differences in pathology and virulence of each parasite.

\section{Effects of Nosema apis infection on worker midgut development}

Our results suggest that $N$. apis infection may impair midgut development at early time points, since genes involved in molecular processes likely associated with repairing tissue damage were significantly regulated. Interestingly, along with genes involved in neurogenesis and tube morphogenesis, we saw significant regulation of headcase [66] and slit [67] which interact with other 
Table 8 Expression patterns of select candidate genes regulating metabolism, development and immunity

\begin{tabular}{|c|c|c|c|c|c|c|}
\hline Function & Candidate genes & AM ID & Flybase ID & $\begin{array}{l}\text { Control: } \\
\text { Nosema }\end{array}$ & $\begin{array}{l}\text { Nurse: } \\
\text { Forager }\end{array}$ & $\begin{array}{l}\text { Rich: } \\
\text { Poor }\end{array}$ \\
\hline \multirow{13}{*}{$\begin{array}{l}\text { Insulin signaling, metabolism } \\
\text { and behavioral maturation }\end{array}$} & Insulin-like peptide 2 & AM00001 & FBgn0044050 & Control & Nursef & Rich $\neq$ \\
\hline & $\overline{\text { PDK1 }}$ & AM08362 & FBgn0020386 & Nosema & Forager & NR \\
\hline & Akt1 & AM08268 & FBgn0010379 & Control & NR & NR \\
\hline & Foxo & $\begin{array}{l}\text { AM06472, } \\
\text { AM04383* }\end{array}$ & FBgn0038197 & Nosema & NR & Poor \\
\hline & Lipase-1 & AM03164 & FBgn0032264 & Control & Nurse & Rich \\
\hline & Lipid storage droplet 2 & AM11728 & FBgn0030608 & Control & Foragerf & NRキ \\
\hline & Cpt1 & AM11065 & FBgn0027842 & Control & Nursef & Rich $\neq$ \\
\hline & Usp & AM09226 & FBgn0003964 & Nosema & NR & NR \\
\hline & Gigas & AM06808 & FBgn0005198 & Control & NR & NR \\
\hline & $\begin{array}{l}\text { Juvenile hormone epoxide } \\
\text { hydrolase }\end{array}$ & AM03396 & FBgn0010053 & Control & Nursef & Rich $\neq$ \\
\hline & Juvenile hormone esterase & AM07915 & FBgn0010052 & Control & Nurse & Rich $\neq$ \\
\hline & Trehalose-6-phosphate synthase 1 & AM05412 & FBgn0027560 & Nosema & Forager & Rich \\
\hline & Venus kinase receptor & AM08479 & No orthologue & Nosema & Forager & NR \\
\hline \multirow[t]{2}{*}{ Immunity } & ECSIT & AM11800 & FBgn0028436 & Control & NR & Rich \\
\hline & Pelle & AM08978 & FBgn0010441 & Control & Forager & Rich \\
\hline
\end{tabular}

*AM04383 was only significantly regulated in this study.

‡Data from qRT-PCR [43].

Directional expression of candidate genes was compared between this study (control:co-infected workers, 14 days pi) and expression in nurses versus foragers and workers fed on rich versus poor diets obtained from microarrays or quantitative real-time PCR [43]. Treatment names in the table indicate upregulation of a given transcript in the assigned treatment group relative to the other experimental group. NR indicates that no significant regulation was identified between groups.

factors to respectively mediate tracheal branching and directional growth in fruit flies. Since the trachea supply tissues with oxygen, these changes may be due to tissue repair mechanisms and/or changing respiratory demands of midgut cells as they begin to support parasite propagation. Indeed, previous studies by Dussabaut and colleagues (2012) examined the impact of $N$. ceranae infection in worker midgut tissue and found that similar GO categories, including tracheal development, were affected at 7 days post-infection (but 14 days of age). Sli was also regulated in this study [28] and incorporated in a larger network of genes involved in tissue regeneration (Wnt signaling pathway).

\section{Effects of Nosema infection on worker immunity}

Previous studies suggest that Nosema infection alters expression of canonical immune genes in abdominal tissues, though this response is modulated by Nosema species, spore load, infection period/age of bee, and genotype of the bee $[32,33,68]$. Similarly, studies examining interactions between bumble bees and their intestinal, trypanosomal parasites have shown that host expression of immune genes is modulated by infection period [69]. Interestingly, in these experiments, genes found responding to acute immunostimulation in a previous study [58], but not canonical immune genes [57] as a group were significantly regulated across all infection types, tissues and timepoints, suggesting that non-canonical immune genes may be important in moderating local and systemic defense responses to Nosema. However, in co-infected workers, we did note changes in select members (e.g. pelle, ECSIT) of the Toll signaling pathway which is thought to defend insects against fungi [56]. Interestingly, members of the Toll signaling pathway were differentially regulated in drones from selected, $N$. ceranae tolerant, Danish lineages and unselected German lineages, suggesting that the Toll signaling pathway may play an active role in honey bee defense against this pathogen [68]. Given the temporally dynamic nature of immune responses, the high degree of cross-talk amongst pathways and the likely role of non-canonical immune genes, future immune studies may track broader suites of candidate genes over longer time courses. Furthermore, local constitutive immune responses, not captured in transcriptional studies, may play a role in defense as suggested by [28].

\section{Effects of Nosema infection on worker fat body metabolism; links to worker nutrition and behavioral maturation}

Consistent with previous studies indicating that infection is energetically costly, we found that Nosema infection broadly altered expression of genes involved in metabolic processes in worker fat body tissue. Metabolic changes were evidenced at earlier ( 2 and 7 day) timepoints, where 
$N$. apis infection impacted lipid metabolism and mitochondrial membrane organization. At later time points in co-infected bees, expression of transcripts related to carbohydrate, protein and lipid metabolism was reduced relative to controls. Previous studies demonstrate that the fat body can direct nutrient-sensitive transitions in worker behavioral maturation. Thus infection-mediated changes in this tissue's metabolism may in part drive documented precocious foraging behavior. Indeed, comparative analyses validated both metabolic and maturational changes in co-infected worker gene expression profiles. Also, genes previously implicated in both worker nutrition and maturation were regulated in this study. Candidates from the insulin signaling pathway that were regulated in this study (Apis mellifera ilp-2; Akt1; Pdk1; foxo, gigas) may be of particular interest for future studies investigating molecular mechanisms behind symptoms of Nosema infection. Current theory suggests that insulin signaling in worker honey bees performs both a conserved role in regulating individuals' nutritional balances and an evolutionarily co-opted role in division of labor by contributing to worker task-orientation [40]. Thus, at the molecular level, changes in the insulin signaling pathway may reflect energetic costs of Nosema infection and reflexively promote changes within the same pathway or linked networks that stimulate precocious foraging.

\section{Modeling the direct and indirect costs of Nosema infection on workers}

Based on these findings, we suggest three possible models (Figure 2) for how Nosema affects worker physiology, immunity and behavioral maturation. First, Nosema infection may directly regulate expression of immune genes. Second, Nosema may 'starve' its hosts through destruction of midgut tissue and/or by appropriating host resources, resulting in accelerated behavioral maturation and associated changes in worker hormones, metabolism and immunity. Third, Nosema may directly impact expression of genes regulating behavioral maturation leading to associated changes in metabolism and immunity. These pathology scenarios are not mutually exclusive and may operate in concert to produce symptoms of infection. To better illuminate the relative contributions of restricted nutrition/impaired metabolism in infected workers versus direct manipulation of gene expression in driving precocious foraging in infected workers, we determined whether gene expression profiles of co-infected workers were more similar to that of bees fed on poor diets or foragers [43]. Results from a Chi-Square test found that infected workers were more similar to bees fed on a poor diet than foragers. Workers were caged in this study and the diet study, but not the behavior study, which may have contributed to overall similarity between this study and the diet study. However, as demonstrated by directional overlap analyses (see Table 5), treatment (infection status or diet) is a primary driver of similarity between studies. Taken together, these analyses, highlight the second model as an important pathway for Nosema disease etiology. Additionally, GO analyses of overlapping transcripts with the same directional expression between this study (14 days pi, co-infected workers) and diet and maturation gene lists both produced significant or near-significant immune categories (Tables 6 and 7). These findings suggest a complex relationship between worker immunity, behavioral state and hormonal and metabolic profiles. Indeed,

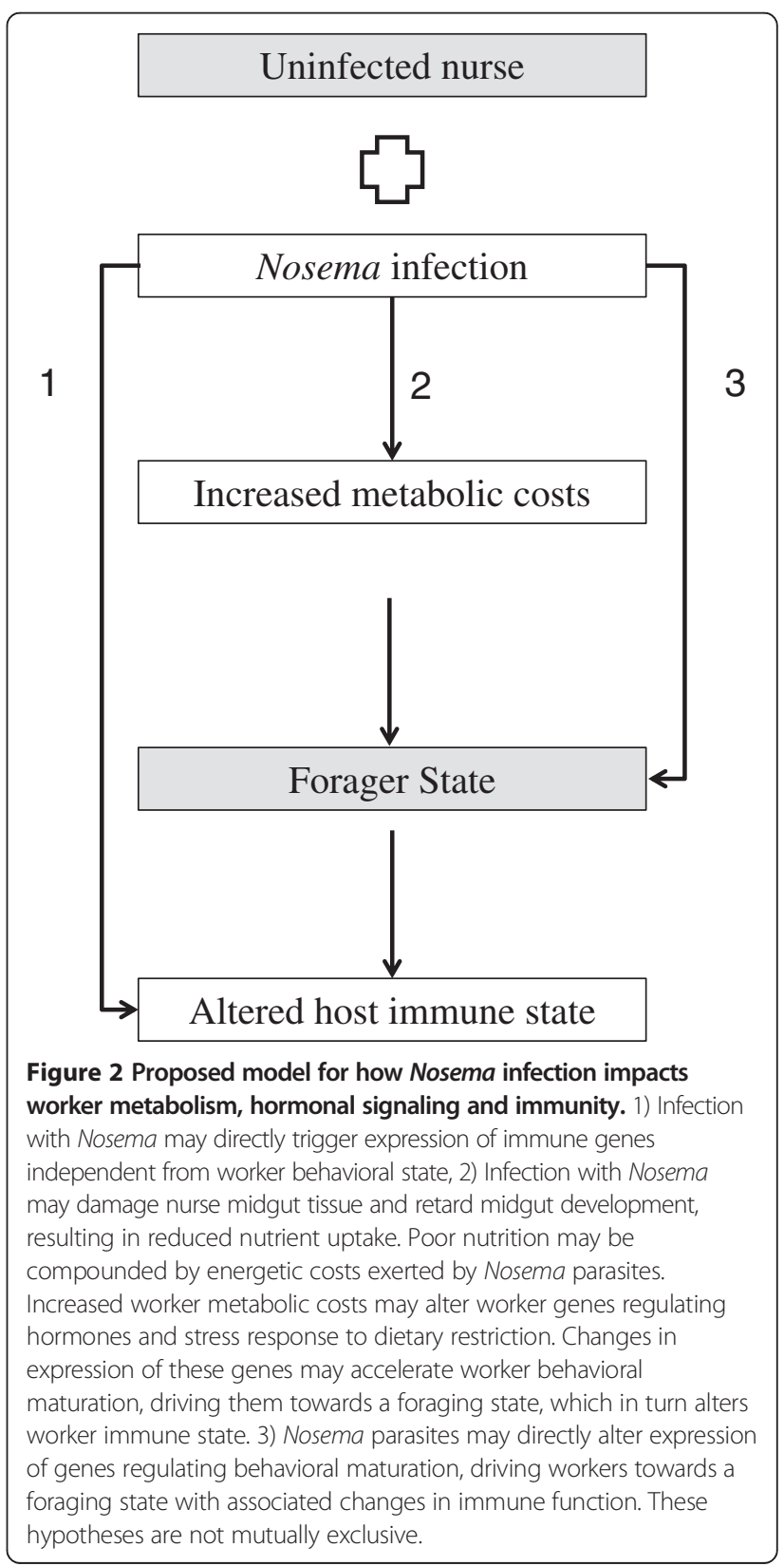


studies in insects and other animals point to the importance of nutrition in optimizing immunity [70]. The impoverished nutritional/metabolic status of infected workers may compromise their immune defenses though additional studies employing secondary pathogen challenges are needed to test this hypothesis.

\section{Common responses to diverse stressors in honey bees}

Worker genomic responses to Nosema infection may also represent common, chronic stress response pathways in honey bees. In mammals, acute stress impels catabolism of energy stores to fuel fight-or-flight responses but chronic stress can lead to dysregulation of the molecular pathways sustaining the stress response [71]. Negative, interlinked consequences of prolonged stress, inflammation and metabolic dysfunction have been well-documented in mammalian models [72,73]. Though additional work is required to characterize responses to immediate and protracted stress in honey bees, several factors (e.g. biogenic amines, neuropeptides, and hormones regulating energy metabolism) have been identified as candidate mediators. In addition, vitellogenin, $\mathrm{JH}$ and the insulin signaling pathway, serving as metabolic regulators of stress responses and/or molecular buffers against stress, in addition to other regulators of division of labor, have also been listed as potential contributing agents [71] Thus, stress, distinct from energetic costs of infection, may simultaneously function as a response to and catalytic driver of the metabolic symptoms of Nosema infection and subsequently lead to changes in worker immunity and task-related behaviors. Indeed, studies examining gene expression in $N$. ceranae infected midgut tissue point to the likely involvement of a canonical stress hormone, CRH-BP (corticotrophin-releasing hormone binding protein) [28].

Precocious foraging as a general, behavioral stress response has been previously noted in honey bees. For example, immune challenge with non-replicating pathogens, such as injection with LPS, induces forager-like gene expression, physiology and behavior in treated workers compared with Ringer-injected controls [74]. Workers subjected to wounding via injection and $\mathrm{CO}_{2}$-treated workers forage sooner than non-injected or untreated controls, suggesting that diverse stressors can be powerful instigators of behavioral maturation [35,48,49]. Parasitization with Varroa mites also results in accelerated behavioral maturation [75] and alters expression of members of the insulin and TOR pathways in addition to other molecular markers associated with precocious foraging [76]. Varroa mite (and associated viral) parasitization depresses worker expression of genes related to metabolism, especially protein metabolism [76] and reduces protein content in adult hemolymph, including Vg [77]. However, additional studies indicate that Varroa and associated viral infections may have complex effects on worker protein content and other nutritional markers in workers [78]. Also, not all parasites universally induce precocious foraging in honey bee hosts [75]. Thus, future studies examining common physiological and behavioral outcomes in honey bees to disparate stressors will enhance our understanding of the stress response in this insect model [71].

Overall, precocious foraging, as a byproduct of energetic stress levied by Nosema infection (previously suggested by $[23,25]$ ) and/or a byproduct of the worker stress response to infection, carries costs for individuals and colonies. As previously outlined [11], because precocious foraging results in premature death, and because Nosema infected workers may have greater levels of extrinsic mortality due to other aberrant behavioral or physiological symptoms of infection, Nosema infected colonies face additional energetic costs of rearing replacement workers, who may also forage prematurely. This may result in weaker colony populations, poor food stores [12] and potentially colony death [79]. However, despite costs associated with premature foraging, colonies may also benefit from reduced within-hive pathogen transmission as suggested by [37]. Further studies integrating changes in individual physiology and behavior and colony regulation of division of labor will enhance our understanding of how social structures allocate resources in response to energetically costly infections.

\section{Conclusions}

Our studies demonstrate the complexity of examining the impacts of chronic disease and parasitization on individuals and social groups. The direct and indirect effects of disease or parasitism can be difficult to disentangle. However, by combining timecourses, tissue-specific analyses and genomic tools it is possible to begin to elucidate the molecular mechanisms that mediate disease symptoms, at the level of the individual and ultimately the social group. In the case of Nosema, the disruption of gut integrity and siphoning of host energy may trigger downstream changes in key, interconnected metabolic and hormonal pathways, resulting in changes in host maturation and, thus, ultimately, productivity and longevity. Through comparative analyses we identified molecular pathways underpinning this response, including the insulin signaling pathway. We also discuss the interlinked roles of energetic stress induced by Nosema parasitism and stress as a host response to infection. Future studies are needed to elucidate the relative contribution of each of these two types of stress to catalyzing disease symptoms. Changes in individual worker maturation as a consequence of infection likely affect both disease transmission dynamics and colony age-structure and organization, thereby impacting colony productivity and health. 


\section{Methods \\ Worker infection}

Honey bee colonies headed by Kona Italian queens (Kona Queen Hawaii, Inc, Captain Cook, HI) were maintained in the Weslaco, TX apiary according to standard beekeeping practices. In 2008, frames of late-stage brood were placed in an incubator at $33^{\circ} \mathrm{C}$ for 24 hours to obtain newly emerged workers. Workers were placed in cages $(12 \times 17 \times 9 \mathrm{~cm})$ with 100 bees per cage. Fifty workers were randomly selected from each cage, restrained, and fed 5 ul of 50\% sugar solution with or without 50,000 Nosema apis spores. Workers were marked and returned to their cage. Cages were maintained in an incubator at $33^{\circ} \mathrm{C}, 50 \% \mathrm{RH}$ and kept in the dark. Bees were fed MegaBee (MegaBee, Yuma, AZ) as a pollen-substitute and protein source and 50\% sugar water ad libitum. Megabee- and pollen-fed caged bees show similar physiological development [80]. Also, rearing Nosema infected bees in cages with supplementary diets has been done previously $[28,32,36]$ and physiological impacts of Nosema infection have been demonstrated in cage and field studies [23-25,32,36,52]. Bees were collected on dry ice at 1,2 and 7 days pi (post-infection) and stored at $-80^{\circ} \mathrm{C}$. Nosema spores were amplified in caged bees prior to the experiment at the USDA laboratory in Weslaco, TX. $N$. apis spores originated from a long standing culture maintained at Kentucky State University.

In 2010, $N$. apis spores were obtained as before and $N$. ceranae spores were isolated from a single bee collected in a heavily infected commercial apiary (LA, USA). Spores of both species were amplified in cages and fed to newly emerged workers as before. This time, however, workers were fed 25,000 spores of $N$. apis and 25,000 spores of $N$. ceranae and were collected on dry ice after 14 days. All samples were stored at $-80^{\circ} \mathrm{C}$.

Samples from both years were shipped to Penn State University on dry ice, and RNA from the migduts of samples collected in 2008 was converted to cDNA and tested for Nosema infection using $N$. apis and $N$. ceranae specific primers (Additional file 2: Table S5) using conventional PCR and reaction conditions as in [81,82]. In 2010, midguts were homogenized in RLT/BME buffer (Allprep DNA/RNA/Protein Mini Kit, Qiagen, Valencia, CA) and an aliquot was taken for DNA extraction. Briefly, 90 ul of each homogenate was incubated overnight with 125 ul of a CTAB buffer (100 mM Tris- $\mathrm{HCl}$ ( $\mathrm{pH} 8.0)$, 20 mM EDTA (pH8.0), 1.4 M NaCl, 2\% Cetyltrimethylammonium bromide, $0.2 \%$ 2-mercaptoethanol) at $56^{\circ} \mathrm{C}$ with $20 \mathrm{ul}$ of proteinase $\mathrm{K}$ from the Allprep kit. (CTAB protocol courtesy of Dr. Judy Chen, USDA-ARS, Beltsville. CTAB buffer breaks down microsporidia spore walls). In the morning, the sample was added to Allprep columns and DNA was extracted. DNA was checked for Nosema spp. infection using conditions in $[81,82]$.

\section{Dissection, RNA extraction and array hybridization}

We thawed individual bees on ice-chilled, sterile plates under cold RNAlater (Qiagen, Valencia, CA) and dissected midguts from bees collected 1 and 2 days pi (postinfection) in 2008. We dissected fat bodies (eviscerated abdomens with digestive and reproductive organs and venom sac removed) at 2 and 7 days pi from bees collected in 2008 and at 14 days pi from bees collected in 2010. We pooled tissues from 5 bees per biological replicate ( $n=4$ per tissue and timepoint).

In 2008, RNA was extracted from midgut and fat body samples with Trizol (Invitrogen, Carlsbad, CA) and amplified with the Ambion MessageAmp II aRNA Amplification Kit (AM1751, Life Technologies, Grand Island, NY). Each sample was labeled independently with $\mathrm{Cy} 3$ or Cy5 using the ULS aRNA fluorescent labeling kit (EA-006, Kreatech, Amsterdam, Netherlands) and hybridized with the Maui Hybridization System (BioMicro Systems, Salt Lake City, UT) to whole genome, oligonucleotide microarrays [83] obtained from the Keck Center for Comparative and Functional Genomics (University of Illinois, Urbana-Champaign, USA). Arrays were scanned with the Axon Genepix 4000B scanner (Molecular Devices, Sunnyvale, CA) and viewed with GENEPIX software (Agilent Technologies, Santa Clara, CA). In 2010, RNA was extracted from fat body samples with the RNeasy Kit (Qiagen, Valencia, CA) and amplified, labeled and hybridized as before. For each tissue and timepoint, we used a loop design incorporating dye swaps that resulted in 16 arrays for midgut tissue, 16 arrays for fat body tissue in 2008 and 8 arrays for fat body tissue in 2010 . Thus each array experiment incorporated 4 biological replicates per treatment and 2 technical replicates per sample.

\section{Microarray data analysis}

The 2008 midguts, 2008 fat bodies, and 2010 fat bodies were analyzed as three separate studies. For all analyses, we removed spots with an intensity less than 100 (the intensity level of the array background) in addition to transcripts with less than 7 observations from each analysis. We log-transformed and normalized expression data using a mixed-model ANOVA in SAS (proc MIXED, Cary, NC):

$$
\begin{aligned}
\mathrm{Y}= & \mu+\text { dye }+ \text { block }+ \text { array }+ \text { array } * \text { dye }+ \text { array } \\
& * \text { block }+\epsilon
\end{aligned}
$$

where $\mathrm{Y}$ is expression, dye and block are a fixed effects, and array, array*dye and array*block are random effects. Genes with significant expression differences between groups were detected by using a mixedmodel ANOVA. 
We used the following model for midguts and fat bodies collected in 2008:

$$
\begin{aligned}
\mathrm{Y}= & \mu+\text { treatment }+ \text { age }+ \text { treatment } * \text { age }+ \text { spot } \\
& + \text { dye }+ \text { array }+\epsilon
\end{aligned}
$$

where Y represents the residual from the previous model. Treatment, age, treatment*age, spot and dye are fixed effects and array is a random effect.

We used the following model for fat bodies collected in 2010:

$$
\mathrm{Y}=\mu+\text { treatment }+ \text { spot }+ \text { dye }+ \text { array }+\epsilon
$$

where Y represents the residual from the previous model. Treatment, spot and dye are fixed effects and array is a random effect.

$p$-values were corrected for multiple testing using a false discovery rate of FDR $<0.001$ for midgut and fat body tissue collected in 2008 (proc MULTTEST, SAS). $p$-values for fat body samples collected in 2010 were corrected for multiple testing using an FDR $<0.0001$. Because a greater number of transcripts were affected in the latter study, we chose a more stringent FDR value, allowing for a more targeted analyses of core genes that were regulated by Nosema co-infection. The array datasets supporting the results of this article are available at the ArrayExpress repository $(N$. apis infection in worker midgut tissue: E-MEXP-3891, N. apis infection and worker fat body tissue: E-MEXP-3892, Co-infection and worker fat body tissue: E-MEXP-3889).

We conducted principal components analysis (JMP 9.0.2, SAS, Cary, NC) to determine relative impact of treatment, age and age $\mathrm{x}$ treatment interactions on gene expression. Genes with a significant treatment effect only were selected for subsequent analyses. Gene ontology (GO) analysis was performed using the Functional Annotation Tool from DAVID (version 6.7) [84,85] with a significance cutoff of $p<0.05$. For all $\mathrm{GO}$ analyses, array transcripts were matched to Flybase orthologs and the entire array transcript list with matches to Flybase orthologs was used as a background list (7,186 unique Flybase genes). Note that the oligos on the arrays were annotated in 2007 [86]. Candidate gene annotations presented in Table 8 were manually annotated by blasting oligo nucleotide probe sequences from the arrays, and then selecting protein sequence of the best honey bee gene match and blasting these protein sequences against the translated Drosophila melanogaster genome. Reciprocal blasts were performed on some genes to check accuracy of identification.

\section{Comparisons with previous studies}

We compared transcripts/genes significantly regulated by infection status with transcripts/gene lists from five other studies, described below.

1. Nosema ceranae genes (midgut tissue) [28]: Genes significantly and differentially regulated in control workers vs workers infected for 7 days with $N$. ceranae.

2. Canonical immune genes [57]: Canonical immune genes identified during annotation of the honey bee genome.

3. Acute immune response transcripts (fat body tissue) [58]: Transcripts significantly and differentially regulated by immune challenge (bacteria-, Sephadex bead-, or saline-injection) in worker bees

4. Nurses versus foragers (fat body tissue) [43]: Significantly, differentially regulated transcripts between nurses and foragers.

5. Workers fed on rich versus poor diets (fat body tissue) [43]: Significantly, differentially regulated transcripts between workers fed on rich (honey and pollen) and poor diets (sugar syrup).

For these study comparisons, note that the transcripts on the microarrays are designated with "AM" (Apis mellifera) numbers [86]. These transcripts correspond to annotated genes from the honey bee genome, which are annotated with "GB" (GBrowse) numbers [87]. For comparisons with gene lists generated from the honey bee arrays (\#3-5), it was possible to directly compare gene lists based on transcript AM numbers. For comparisons with other studies (\#1-2) which investigated whole genome data but did not use microarrays or used tiling arrays, it was necessary to use GB annotations for microarray transcripts rather than AM notations. We identified overlap between studies using an online Venn diagram service [88] and tested for significance using a hypergeometric test [89]. Background overlap of between studies was assumed to be either the entire list of AM numbers (studies \#3-5) or GB numbers on the arrays (\#1-2). Select GO analyses based on study overlap were performed in DAVID as above.

\section{Validation of gene expression using quantitative real-time $P C R$}

We examined gene expression levels of three antimicrobial peptides involved in immune function (abaecin, defensin-1, hymenoptaecin) [57] and expression of two additional genes involved in worker maturation and/or nutrition (vitellogenin [45], hexamerin $70 b$ [90]). We used the total RNA extracted from worker fat bodies for the microarray analysis and compared gene expression between bees with Nosema apis infections and controls at 7 days pi, and gene expression between bees with mixed Nosema infections 
and controls at 14 days pi. cDNA was synthesized using SuperScript II First-Strand Synthesis System for RT-PCR (Invitrogen-Life Technologies, Carlsbad, CA, USA) and oligo-dT primers according to the manufacturer's protocol. The cDNA was then diluted $10(\mathrm{x})$ with double distilled autoclaved water. Amplification was performed in a $10 \mu \mathrm{l}$ reaction mixture containing reaction mix of $0.5 \mathrm{U}$ of GoTaqR Flexi DNA polymerase (Promega Co., Madison, WI) with the colorless $5 \times$ GoTaqR Flexi buffer, $0.38 \mathrm{mM}$ dNTP mix, $5.0 \mathrm{mM} \mathrm{MgCl}_{2}, 0.35 \mu \mathrm{M}$ of each primer, $0.33 \mu \mathrm{l}$ of a 1/1000 stock dilution of SYBR-Green (Invitrogen Corp.), and $1 \mu \mathrm{l}$ of cDNA. Reactions were loaded on the CFX96 $^{\text {ma }}$ Real-Time PCR Detection System (BioRad Ltd, Hercules, CA) and run for 40 cycles at $95^{\circ} \mathrm{C}(5 \mathrm{~s})$ and $60^{\circ} \mathrm{C}$ (30 s) after initial denaturing at $95^{\circ} \mathrm{C}$ for 3 minutes. Fluorescence was measured at the end of the annealing stage of every cycle. A final extension of $72^{\circ} \mathrm{C}(2 \mathrm{mins})$ followed by a melting curve analysis was added to the program to ensure that true product was being amplified. Negative control reactions were included in each run and contained all reaction components except the template. This helped discern any primer dimers that may have amplified. Individual samples were standardized against actin expression levels. Primers used for these genes were previously published [78] and can be found in Additional file 2: Table S6. Relative fold expression of candidate genes between treatment groups was calculated and differences in gene expression were tested for significance with Mann Whitney U Tests in JMP 10 (Cary, NC).

\section{Additional files}

Additional file 1: Figure S1-S3. This file includes supplementary figures documenting PCR results, directional regulation of transcripts within significant GO categories and GRT-PCR results.

Additional file 2: Tables S1-S6. Supplementary Tables 1-6 include lists of significantly regulated transcripts identified in this study as well as transcript annotation. These tables also include data about primers used for study validation and results of qRT-PCR analyses.

\section{Abbreviations}

JH: Juvenile hormone; vg: Vitellogenin; pi: Post-infection; GO: Gene ontology; IMD: Immunodeficiency; JAK/Stat: Janus kinase/signal transducer and activator of transcription; ECSIT: Evolutionarily conserved signaling intermediate in toll pathway; Amllp-2: Apis mellifera insulin-like peptide 2; Pdk1: 3-phosphoinositide-dependent protein kinase 1; foxo: Forkhead box protein O; Akt: Serine/threonine protein kinase; Jhe: Juvenile hormone esterase; Jheh: Juvenile hormone epoxide hydrolase; Usp: Ultraspiracle; NF-kB: Nuclear factor kappa B; JNK: c-Jun amino-terminal kinase; AMP: Antimicrobial peptide; Cpt1: Carnitine O-palmitoyl transferase 1; Isd-2: Lipid storage droplet 2; TOR: Target of rapamycin; VKR: Venus kinase receptor; NR: Not regulated; CRH-BP: Corticotrophin-releasing hormone binding protein.
\end{abstract}

\section{Competing interests}

The authors declare no competing interests.

\section{Authors' contributions}

$\mathrm{HLH}, \mathrm{KAA}$ and CMG designed the study and interpreted the results. KAA conducted infection experiments, collected samples and conducted qRT-PCR.
HLH performed the microarray experiments and analyzed data. All authors wrote, read and approved the final manuscript.

\section{Acknowledgements}

We would like to thank Dr. Thomas Webster for provision of Nosema spores, Bob Cox for expert beekeeping assistance, Kevin Donohue for assistance with RNA extractions, and Eduardo Saldivar for assistance with PCR and qRT-PCR verification of Nosema infection and candidate gene expression levels. HLH was supported by a University Fellowship from Pennsylvania State University and the Häagan Dazs Fellowship in Honey Bee Health from the Department of Entomology at Pennsylvania State University. This material is also based upon work supported by the National Science Foundation under Grant No. DGE1255832 to HLH. Any opinions, findings, and conclusions or recommendations expressed in this material are those of the authors and do not necessarily reflect the views of the National Science Foundation. This research was also supported by the USDA-CAP Managed Pollinator Grant to KAA and CMG and additional funding from USDA-AFRI (2009-05207) to C.M.G.

\section{Author details}

'Department of Entomology, Center for Pollinator Research, Center for Chemical Ecology, Huck Institutes of the Life Sciences, Pennsylvania State University, University Park, USA. ${ }^{2}$ Honey Bee Breeding, Genetics \& Physiology Lab, USDA, Baton Rouge, USA. ${ }^{3}$ A Chemical Ecology Laboratory,

Pennsylvania State University, Orchard Road, University Park, PA 16802, USA.

Received: 11 April 2013 Accepted: 7 November 2013

Published: 18 November 2013

\section{References}

1. Afonso C, Paixão VB, Costa RM: Chronic toxoplasma infection modifies the structure and the risk of host behavior. PLoS One 2012, 7(3):e32489.

2. Cremer S, Armitage SAO, Schmid-Hempel P: Social immunity. Curr Biol 2007, 17(16):R693-R702.

3. Fries I: Nosema ceranae in European honey bees (Apis mellifera). J Invertebr Pathol 2010, 103:S73-S79.

4. Williams BAP: Unique physiology of host-parasite interactions in microsporidia infections. Cellular microbiology 2009, 11(11):1551-1560

5. Gisder S, Möckel N, Linde A, Genersch E: A cell culture model for Nosema ceranae and Nosema apis allows new insights into the life cycle of these important honey bee-pathogenic microsporidia. Environmental microbiology 2011, 13(2):404-413.

6. Wilson-Rich N, Spivak M, Fefferman NH, Starks PT: Genetic, individual, and group facilitation of disease resistance in insect societies. Annu Rev Entomol 2009, 54(1):405-423.

7. Paxton RJ, Klee J, Korpela S, Fries I: Nosema ceranae has infected Apis mellifera in Europe since at least 1998 and may be more virulent than Nosema apis. Apidologie 2007, 38(6):558.

8. Higes M, Garcia-Palencia P, Martin-Hernandez R, Meana A: Experimental infection of Apis mellifera honeybees with Nosema ceranae (Microsporidia). J Invertebr Pathol 2007, 94(3):211-217.

9. Managed Pollinator Coordinated Agricultural Project, Objective 1.1. [http://www.beeccdcap.uga.edu/objectives/obj11.html].

10. Forsgren E, Fries I: Comparative virulence of Nosema ceranae and Nosema apis in individual European honey bees. Vet Parasitol 2010, 170(3-4):212-217.

11. Higes M, Martín-Hernández R, Meana A: Nosema ceranae in Europe: an emergent type C nosemosis. Apidologie 2010, 41(3):375-392.

12. Botias C, Martin-Hernandez R, Barrios L, Meana A, Higes M: Nosema spp. infection and its negative effects on honey bees (Apis mellifera iberiensis) at the colony level. Vet Res 2013, 44(1):25.

13. Fenoy S, Rueda C, Higes M, Martín-Hernández R, del Aguila C: High-level resistance of Nosema ceranae, a parasite of the honeybee, to temperature and desiccation. Applied and environmental microbiology 2009, 75(21):6886-6889.

14. Gisder S, Hedtke K, Mockel N, Frielitz MC, Linde A, Genersch E: Five-year cohort study of Nosema spp. in Germany: does climate shape virulence and assertiveness of Nosema ceranae? Applied and environmental microbiology 2010, 76(9):3032-3038.

15. Martin-Hernandez R, Meana A, Garcia-Palencia P, Marin P, Botias C, Garrido-Bailon E, Barrios L, Higes M: Effect of temperature on the biotic 
potential of honeybee microsporidia. App/ Environ Microbiol 2009 75(8):2554-2557.

16. Alaux C, Brunet J, Dussaubat C, Mondet F, Tchamitchan S, Cousin M: Interactions between Nosema microspores and a neonicotinoid weaken honeybees (Apis mellifera). Environmental microbiology 2010, 12(3):774-782.

17. Wu JY, Smart MD, Anelli CM, Sheppard WS: Honey bees (Apis mellifera) reared in brood combs containing high levels of pesticide residues exhibit increased susceptibility to Nosema (Microsporidia) infection. J Invertebr Pathol 2012, 109(3):326-329.

18. Huang W-F, Solter LF, Yau PM, Imai BS: Nosema ceranae escapes fumagillin control in honey bees. PLoS Pathog 2013, 9(3):e1003185.

19. Pettis JS, van Engelsdorp D, Johnson J, Dively G: Pesticide exposure in honey bees results in increased levels of the gut pathogen Nosema. Naturwissenschaften 2012, 99(2):153-158.

20. Liu TP: Ultrastructure of the midgut of the worker honey bee (Apis mellifera) heavily infected with Nosema apis. J Invertebr Pathol 1984, 44(3):282-291.

21. Chen YP, Evans JD, Murphy C, Gutell R, Zuker M, Gundensen-Rindal D, Pettis JS: Morphological, molecular, and phylogenetic characterization of Nosema ceranae, a microsporidian parasite isolated from the European honey bee, Apis mellifera. J Eukaryot Microbio/ 2009, 56(2):142-147.

22. Cornman RS, Chen YP, Schatz MC, Street C, Zhao Y, Desany B, Egholm M Hutchison S, Pettis JS, Lipkin Wl, et al: Genomic analyses of the Microsporidian Nosema ceranae, an emergent pathogen of honey bees. PLOS Pathog 2009, 5(6):14

23. Mayack C, Naug D: Energetic stress in the honeybee Apis mellifera from Nosema ceranae infection. J Invertebr Pathol 2009, 100(3):185-188.

24. Martín-Hernández R, Botías C, Barrios L, Martínez-Salvador A, Meana A, Mayack C, Higes M: Comparison of the energetic stress associated with experimental Nosema ceranae and Nosema apis infection of honeybees (Apis mellifera). Parasitology research 2011, 109(3):605-612

25. Mayack C, Naug D: Parasitic infection leads to decline in hemolymph sugar levels in honeybee foragers. J Insect Physiol 2010, 56(11):1572-1575.

26. Naug D, Gibbs A: Behavioral changes mediated by hunger in honeybees infected with Nosema ceranae. Apidologie 2009, 40(6):595-599.

27. Wang D-I, Moeller FE: Comparison of the free amino acid composition in the hemolymph of healthy and Nosema-infected female honey bees. J Invertebr Pathol 1970, 15(2):202-206.

28. Dussaubat C, Brunet J-L, Higes M, Colbourne JK, Lopez J, Choi J-H, Martín-Hernández R, Botías C, Cousin M, McDonnell C, et al: Gut pathology and responses to the Microsporidium Nosema ceranae in the honey bee Apis mellifera. PLoS One 2012, 7(5):e37017.

29. Malone LA, Gatehouse HS: Effects of Nosema apis infection on honey bee (Apis mellifera) digestive proteolytic enzyme activity. J Invertebr Patho 1998, 71(2):169-174.

30. De Graaf DC, Raes H, Sabbe G, De Rycke PH, Jacobs FJ: Early cevelopment of Nosema apis (Microspora: Nosematidae) in the midgut epithelium of the honeybee (Apis mellifera). J Invertebr Pathol 1994, 63(1):74-81.

31. Paldi N, Glick E, Oliva M, Zilberberg Y, Aubin L, Pettis J: Effective gene silencing in a Microsporidian parasite associated with honeybee (Apis mellifera) colony declines. Applied and environmental microbiology 2010, 76(17):5960-5964.

32. Antunez K, Martin-Hernandez R, Prieto L, Meana A, Zunino P, Higes M: Immune suppression in the honey bee (Apis mellifera) following infection by Nosema ceranae (Microsporidia). Environmental microbiology 2009, 11(9):2284-2290.

33. Chaimanee V, Chantawannakul P, Chen Y, Evans JD, Pettis JS: Differential expression of immune genes of adult honey bee (Apis mellifera) afte inoculated by Nosema ceranae. J Insect Physiol 2012, 58(8):1090-1095.

34. Hassanein MH: The influence of infection with Nosema apis on the activities and longevity of the worker honeybee. Ann Appl Biol 1953, 40(2):418-423.

35. Woyciechowski M, Moroń D: Life expectancy and onset of foraging in the honeybee (Apis mellifera). Insectes sociaux 2009, 56(2):193-201.

36. Goblirsch $M$, Huang ZY, Spivak M: Physiological and behavioral changes in honey bees (Apis mellifera) induced by Nosema ceranae infection. PLoS One 2013, 8(3):6.

37. Dussaubat C, Maisonnasse A, Crauser D, Beslay D, Costagliola G Soubeyrand S, Kretzchmar A, Le Conte Y: Flight behavior and pheromone changes associated to Nosema ceranae infection of honey bee workers (Apis mellifera) in field conditions. J Invertebr Pathol 2013, 113(1):42-51.
38. Wang D, Moeller F: The division of labor and queen attendance behavior of Nosema-infected worker honeybees. J Econ Entomol 1970, 63:1539-1541.

39. Page RE Jr, Peng CYS: Aging and development in social insects with emphasis on the honey bee, Apis mellifera L. Exp Gerontol 2001, 36(4-6):695-711.

40. Ament SA, Wang Y, Robinson GE: Nutritional regulation of division of labor in honey bees: toward a systems biology perspective. Wiley Interdiscip Rev Syst Biol Med 2010, 2(5):566-576.

41. Slessor $\mathrm{K}$, Winston $M$, Conte $\mathrm{Y}$ : Pheromone communication in the honeybee (Apis mellifera L.). J Chem Ecol 2005, 31(11):2731-2745.

42. Toth AL, Kantarovich S, Meisel AF, Robinson GE: Nutritional status influences socially regulated foraging ontogeny in honey bees. J Exp Biol 2005, 208(24):4641-4649.

43. Ament SA, Chan QW, Wheeler MM, Nixon SE, Johnson SP, Rodriguez-Zas SL, Foster LJ, Robinson GE: Mechanisms of stable lipid loss in a social insect. J Exp Biol 2011, 214(22):3808-3821.

44. Toth $\mathrm{AL}$, Robinson GE: Worker nutrition and division of labour in honeybees. Anim Behav 2005, 69:427-435.

45. Amdam GV, Omholt SW: The hive bee to forager transition in honeybee colonies: the double repressor hypothesis. J Theor Biol 2003, 223(4):451-464.

46. Robinson $\mathrm{G}$ : Regulation of honey bee age polyethism by juvenile hormone. Behav Ecol Sociobiol 1987, 20(5):329-338.

47. Marco Antonio D, Guidugli-Lazzarini K, Nascimento A, Simões Z, Hartfelder K: RNAi-mediated silencing of vitellogenin gene function turns honeybee (Apis mellifera) workers into extremely precocious foragers. Naturwissenschaften 2008, 95(10):953-961.

48. Nelson CM, Ihle KE, Fondrk MK, Page RE Jr, Amdam GV: The gene vitellogenin has multiple coordinating effects on social organization. PLOS Biol 2007, 5(3):e62

49. Nilsen KA, Ihle KE, Frederick K, Fondrk MK, Smedal B, Hartfelder K, Amdam GV: Insulin-like peptide genes in honey bee fat body respond differently to manipulation of social behavioral physiology. J Exp Biol 2011, 214(9):1488-1497.

50. Ament SA, Corona M, Pollock HS, Robinson GE: Insulin signaling is involved in the regulation of worker division of labor in honey bee colonies. Proc Natl Acad Sci USA 2008, 105(11):4226-4231.

51. Wang Y, Brent CS, Fennern E, Amdam GV: Gustatory perception and fat body energy metabolism are jointly affected by vitellogenin and juvenile hormone in honey bees. PLoS Genet 2012, 8(6):28.

52. Ares AM, Nozal MJ, Bernal JL, Martin-Hernandez R, Higes M, Bernal J: Liquid chromatography coupled to ion trap-tandem mass spectrometry to evaluate juvenile hormone III levels in bee hemolymph from Nosema spp. infected colonies. J Chromatogr B Analyt Technol Biomed Life Sci 2012 899:146-153.

53. Wilson-Rich N, Dres ST, Starks PT: The ontogeny of immunity: development of innate immune strength in the honey bee (Apis mellifera). J Insect Physiol 2008, 54(10-11):1392-1399.

54. Laughton AM, Boots M, Siva-Jothy MT: The ontogeny of immunity in the honey bee, Apis mellifera L. following an immune challenge. J Insect Physiol 2011, 57(7):1023-1032.

55. Arrese EL, Soulages JL: Insect fat body: energy, metabolism, and regulation. Annu Rev Entomol 2010, 55(1):207-225.

56. Lemaitre B, Hoffman J: The host defense of Drosophila melanogaster. Annu Rev Immunol 2007, 25(1):697-743.

57. Evans JD, Aronstein K, Chen YP, Hetru C, Imler JL, Jiang H, Kanost M Thompson GJ, Zou Z, Hultmark D: Immune pathways and defence mechanisms in honey bees Apis mellifera. Insect Mol Biol 2006, 15(5):645-656

58. Richard F-J, Holt H, Grozinger CM: Effects of immunostimulation on social behavior, chemical communication and genome-wide gene expression in honey bee workers (Apis mellifera). BMC Genomics 2012, 13(1):558.

59. Klee J, Besana AM, Genersch E, Gisder S, Nanetti A, Tam DQ, Chinh TX, Puerta F, Ruz JM, Kryger $P$, et al: Widespread dispersal of the microsporidian Nosema ceranae, an emergent pathogen of the western honey bee, Apis mellifera. J Invertebr Pathol 2007, 96(1):1-10.

60. Wu Q, Brown MR: Signaling and function of insulin-like peptides in insects In: Annual Review of Entomology 2006, 51:1-24.

61. Fingar DC, Blenis J: Target of rapamycin (TOR): an integrator of nutrient and growth factor signals and coordinator of cell growth and cell cycle progression. Oncogene 2004, 23(18):3151-3171. 
62. Ahier A, Rondard P, Gouignard N, Khayath N, Huang S, Trolet J, Donoghue DJ, Gauthier M, Pin J-P, Dissous C: A new family of receptor tyrosine kinases with a venus flytrap binding domain in insects and other invertebrates activated by amino acids. PLoS One 2009, 4(5):e5651.

63. Ament SA, Wang Y, Chen C-C, Blatti CA, Hong F, Liang ZS, Negre N, White KP, Rodriguez-Zas SL, Mizzen CA, et al: The transcription factor Ultraspiracle influences honey bee social behavior and behavior-related gene expression. PLoS Genet 2012, 8(3):e1002596.

64. Mackert A, do Nascimento AM, Bitondi MMG, Hartfelder K, Simões ZLP: Identification of a juvenile hormone esterase-like gene in the honey bee, Apis mellifera L. - expression analysis and functional assays. Comp Biochem Physiol B Biochem Mol Biol 2008, 150(1):33-44.

65. Mackert A, Hartfelder K, Bitondi MMG, Simões ZLP: The juvenile hormone $\mathrm{JH})$ epoxide hydrolase gene in the honey bee (Apis mellifera) genome encodes a protein which has negligible participation in $\mathrm{JH}$ degradation. J Insect Physiol 2010, 56(9):1139-1146.

66. Steneberg P, Samakovlis C: A novel stop codon readthrough mechanism produces functional headcase protein in Drosophila trachea. EMBO Rep 2001, 2(7):593-597.

67. Englund C, Steneberg P, Falileeva L, Xylourgidis N, Samakovlis C: Attractive and repulsive functions of slit are mediated by different receptors in the Drosophila trachea. Development 2002, 129(21):4941-4951.

68. Huang Q, Kryger P, Le Conte Y, Moritz RFA: Survival and immune response of drones of a Nosemosis tolerant honey bee strain towards $N$. ceranae infections. J Invertebr Pathol 2012, 109(3):297-302.

69. Riddell CE, Sumner S, Adams S, Mallon EB: Pathways to immunity: temporal dynamics of the bumblebee (Bombus terrestris) immune response against a trypanosomal gut parasite. Insect Mol Biol 2011, 20(4):529-540.

70. Ponton F, Wilson K, Cotter SC, Raubenheimer D, Simpson SJ: Nutritional immunology: a multi-dimensional approach. PLoS Pathog 2011, 7(12):e1002223.

71. Even N, Devaud J-M, Barron A: General stress responses in the honey bee. Insects 2012, 3(4):1271-1298.

72. Wellen KE, Hotamisligil GS, XF, Khan S: Inflammation, stress, and diabetes. J Clin Invest 2005, 115(5):1111-1119.

73. Black PH: The inflammatory response is an integral part of the stress response: Implications for atherosclerosis, insulin resistance, type II diabetes and metabolic syndrome X. Brain Behav Immun 2003, 17(5):350-364.

74. Alaux C, Kemper N, Kretzschmar A, Le Conte Y: Brain, physiological and behavioral modulation induced by immune stimulation in honeybees (Apis mellifera): a potential mediator of social immunity? Brain Behav Immun 2012, 26(7):1057-1060.

75. Downey $\mathrm{DL}$, Higo TT, Winston ML: Single and dual parasitic mite infestations on the honey bee, Apis mellifera. Insectes sociaux 2000, 47(2):171-176.

76. Alaux C, Dantec C, Parrinello H, Le Conte Y: Nutrigenomics in honey bees: digital gene expression analysis of pollen's nutritive effects on healthy and varroa-parasitized bees. BMC Genomics 2011, 12(1):496.

77. Amdam GV, Hartfelder K, Norberg K, Hagen A, Omholt SW: Altered physiology in worker honey bees (Hymenoptera: Apidae) infested with the mite Varroa destructor (Acari: Varroidae): a factor in colony loss during overwintering? J Econ Entomol 2004, 97(3):741-747.

78. Aronstein KA, Saldivar E, Vega R, Westmiller S, Douglas AE: How Varroa parasitism affects the immunological and nutritional status of the honey bee, Apis mellifera. Insects 2012, 3(3):601-615.

79. Khoury DS, Myerscough MR, Barron AB: A quantitative model of honey bee colony population dynamics. PLoS One 2011, 6(4):e18491.

80. DeGrandi-Hoffman $\mathrm{G}$, Chen $\mathrm{Y}$, Huang $\mathrm{E}$, Huang $\mathrm{MH}$ : The effect of diet on protein concentration, hypopharyngeal gland development and virus load in worker honey bees (Apis mellifera L.). J Insect Physiol 2010, 56(9):1184-1191.

81. Aronstein KA, Saldivar E, Webster TC: Evaluation of Nosema ceranae spore-specific polyclonal antibodies. J Apic Res 2011, 50(2):145-151.

82. Webster TC, Pomper K, Hunt G, Thacker EM, Jones SC: Nosema apis infection in worker and queen Apis mellifera. Apidologie 2004, 35(1):49.

83. Array Express: Honey bee oligo. [http://www.ebi.ac.uk/arrayexpress/arrays/AMEXP-755/]

84. da Huang W, Sherman BT, Lempicki RA: Systematic and integrative analysis of large gene lists using DAVID bioinformatics resources. Nat Protoc 2009, 4(1):44-57.
85. da Huang W, Sherman BT, Lempicki RA: Bioinformatics enrichment tools: paths toward the comprehensive functional analysis of large gene lists. Nucleic Acids Res 2009, 37(1):1-13.

86. Roy J: Carver Biotechnology Center. [http://www.biotech.uiuc.edu/centers].

87. Munoz-Torres MC, Reese JT, Childers CP, Bennett AK, Sundaram JP, Childs KL, Anzola JM, Milshina N, Elsik CG: Hymenoptera genome database: integrated community resources for insect species of the order Hymenoptera. Nucleic Acids Res 2011, 39(suppl 1):D658-D662.

88. Oliveros JC: VENNY: an interactive tool for comparing lists with Venn Diagrams. [http://bioinfogp.cnb.csic.es/tools/venny/index.html].

89. Lund J: Microarray analysis tools: statistical significance of the overlap between two groups of genes. [http://nemates.org/MA/].

90. Cunha AD, Nascimento AM, Guidugli KR, Simões ZLP, Bitondi MMG: Molecular cloning and expression of a hexamerin cDNA from the honey bee, Apis mellifera. J Insect Physiol 2005, 51(10):1135-1147.

doi:10.1186/1471-2164-14-799

Cite this article as: Holt et al:: Chronic parasitization by Nosema microsporidia causes global expression changes in core nutritional, metabolic and behavioral pathways in honey bee workers (Apis mellifera). BMC Genomics 2013 14:799.

\section{Submit your next manuscript to BioMed Central and take full advantage of:}

- Convenient online submission

- Thorough peer review

- No space constraints or color figure charges

- Immediate publication on acceptance

- Inclusion in PubMed, CAS, Scopus and Google Scholar

- Research which is freely available for redistribution 